\title{
Challenges in the Treatment of Gastroesophageal Cancer: Reply
}

\author{
Claire L. Donohoe $\cdot$ John V. Reynolds
}

Published online: 16 March 2011

(c) Société Internationale de Chirurgie 2011

We welcome the comments of Professor Liakakos [1] in respect of our series of 520 patients with adenocarcinoma of the esophagogastric junction (AEG) [2]. The series indicates that neither AEG type nor the operative approach was associated with oncologic outcome by multivariate analysis, and it strongly supports the need to achieve an R0 resection irrespective of operative approach. The more favorable pathologic features and improved outcomes of the predominantly Barrett's-associated AEG type 1, compared with AEG type 2 and AEG type 3, suggests either an earlier diagnosis or altered biology. This observation sets hypotheses for further prospective study with optimal staging in combination with molecular studies.

Professor Liakakos disagrees with our perioperative chemotherapy approach for AEG type 3 tumors, which was either postoperative chemoradiation as per Macdonald et al. [3] or, following the publication of the MAGIC trial [4], preoperative and postoperative chemotherapy as per that regimen, both approaches providing level I evidence from randomized trials and associated with an approximate $25 \%$ improved survival compared with surgery alone. In a Japanese population, a trial by the Adjuvant Chemotherapy Trial of TS-1 for Gastric Cancer (ACTS-GC) group reported superiority of adjuvant S1 compared with surgery alone, and this remains to be tested in a Western population [5]. Professor Liakakos advocates surgery followed by postoperative capecitabine/cisplatin, stating that this is what many surgeons prefer. This may be so, and we agree that this approach may be interesting to study in a trial of

C. L. Donohoe · J. V. Reynolds $(\bowtie)$

Department of Surgery, Trinity College Dublin/St James'

Hospital, Trinity Centre for Health Sciences, St James' Hospital,

Dublin 8, Ireland

e-mail: reynoljv@tcd.ie early stage gastric cancer (IB, II, IIIA) versus surgery alone. However, this approach cannot be recommended as standard, based on existing data.

Finally, as regards the interesting data that have emerged from the use of trastuzumab in the ToGA trial [6], we agree that this offers a intriguing new dimension to the treatment of inoperable, metastatic, or recurrent gastric and junctional tumors that overexpress HER2. There are no indications yet, however, for the use of trastuzumab in the neoadjuvant or adjuvant setting outside of randomized trials, the recruitment, based on power calculations, and planning for which will be daunting in view of the low incidence, approximately $20 \%$, of tumors.

\section{References}

1. Liakakos T (2011) Challenges in the treatment of gastroesophageal junction cancer. World J Surg. doi:10.1007/s00268-011-0977-y

2. Reynolds JV, Ravi N, Muldoon C et al (2010) Differential pathologic variables and outcomes across the spectrum of adenocarcinoma of the esophagogastric junction. World J Surg 34:2821-2829

3 Macdonald JS, Smalley SR, Benedetti J et al (2001) Chemoradiotherapy after surgery compared with surgery alone for adenocarcinoma of the stomach or gastroesophageal junction. N Engl J Med 345:725-730

4. Cunningham D, Allum WH, Stenning SP et al (2006) Perioperative chemotherapy versus surgery alone for resectable gastroesophageal cancer. N Engl J Med 355:11-20

5. Sakuramoto S, Sasako M, Yamaguchi T et al (2007) Adjuvant chemotherapy for gastric cancer with $\mathrm{S}-1$, an oral fluoropyrimidine. N Engl J Med 357:1810-1820

6. Bang Y-J, van Cutsem E, Feyereislova A et al (2010) Trastuzumab in combination with chemotherapy versus chemotherapy alone for treatment of HER2-positive advanced gastric or gastro-oesophageal junction cancer (ToGA): a phase 3, open-label, randomised controlled trial. Lancet 376:687-697 